\title{
SPIN - A Schrödinger-Poisson Solver Including Nonparabolic Bands
}

\author{
H. KOSINA* and C. TROGER \\ Institute for Microelectronics, TU Vienna, Gusshausstrasse 27-29, A-1040 Vienna, Austria
}

\begin{abstract}
Nonparabolicity effects in two-dimensional electron systems are quantitatively analyzed. A formalism has been developed which allows to incorporate a nonparabolic bulk dispersion relation into the Schrödinger equation. As a consequence of nonparabolicity the wave functions depend on the in-plane momentum. Each subband is parametrized by its energy, effective mass and a subband nonparabolicity coefficient. The formalism is implemented in a one-dimensional Schrödinger-Poisson solver which is applicable both to silicon inversion layers and heterostructures.
\end{abstract}

Keywords: Two-dimensional electron gas, heterostructures, MOS capacitor, inversion layer, Schroedinger-Poisson solver, self-consistent

\section{INTRODUCTION}

To accurately model the high-field transport in silicon inversion layers, several authors $[3,4]$ have introduced a nonparabolicity correction in the subband dispersions. In this work we quantitatively analyze nonparabolicity effects in various two-dimensional electron gases. For this purpose a self-consistent Schrödinger-Poisson solver has been developed, capable of dealing with silicon inversion layers and heterostructures. For heterostructures, position-dependent material parameters are taken into account. As a result each subband is characterized by three parameters, $E_{n}$, $m_{n}, \alpha_{n}$, which denote the subband energy, mass and nonparabolicity coefficient, respectively. This set of parameters is intended to serve as input for high-field transport calculations.

Our approach relies on the effective-mass approximation which is applicable if the confining potential, $V(z)$, satisfies two conditions [3]:

1. $V(z)$ is slowly varying over a unit cell,

2. matrix elements of $V(z)$ between Bloch functions of different bands are negligible.

\section{SILICON INVERSION LAYERS}

Within the effective-mass approximation a onedimensional Schrödinger equation can be derived

*Corresponding author: Tel.: +43/1/58801-3719, Fax: +43/1/5059224, e-mail: kosina@iue.tuwien.ac.at. 
from the three-dimensional one provided that the potential only varies in one dimension.

$$
\left(\varepsilon\left(-i \frac{\partial}{\partial z} ; K\right)+V(z)\right) \zeta_{n}(z ; K)=E_{n}(K) \zeta_{n}(z ; K)
$$

In this equation, $\zeta_{n}$ denotes the envelope function, $K$ is the in-plane wave number, and $E(K)$ represents the in-plane dispersion relation. The bulk dispersion relation, $\varepsilon(\boldsymbol{k})$, is assumed to have ellipsoids as equi-energy surfaces, $\varepsilon(\boldsymbol{k})=\varepsilon\left(K^{2} /\right.$ $m_{x y}+k_{z}^{2} / m_{z}$ ). In principle, (1) can be solved numerically for different values of $K$ so as to obtain a point-wise representation of $E(K)$. However, since the bulk dispersion is given by an analytic function one usually is interested in obtaining analytic subband dispersions as well. The latter can be found by applying perturbation theory at $K=0$. The kinetic energy operator is expanded into a Taylor series, and terms up to $K^{4}$ are retained in order to get information on the subband nonparabolicity.

$$
\varepsilon\left(\frac{K^{2}}{m_{x y}}-\frac{1}{m_{z}} \frac{\partial^{2}}{\partial z^{2}}\right) \approx \boldsymbol{T}_{0}+\boldsymbol{T}_{1} K^{2}+\boldsymbol{T}_{2} K^{4}
$$

The unperturbed problem is defined by $\boldsymbol{T}_{0}$, and the terms containing the in-plane wave number are considered as perturbation. The operators $\boldsymbol{T}_{i}$ are given by:

$$
\begin{aligned}
& \boldsymbol{T}_{0}=\varepsilon\left(-\frac{1}{m_{z}} \frac{\partial^{2}}{\partial z^{2}}\right) \\
& \boldsymbol{T}_{1}=\frac{1}{m_{x y}} \cdot \varepsilon^{\prime}\left(-\frac{1}{m_{z}} \frac{\partial^{2}}{\partial z^{2}}\right) \\
& \boldsymbol{T}_{2}=\frac{1}{2 m_{x y}^{2}} \cdot \varepsilon^{\prime \prime}\left(-\frac{1}{m_{z}} \frac{\partial^{2}}{\partial z^{2}}\right)
\end{aligned}
$$

\section{HETEROSTRUCTURES}

For heterostructures we assume nonparabolic dispersion relations for the different semiconduc- tors. The usual implicit definition of the kinetic energy can be generalized to a kinetic energy operator, even when the parameters are positiondependent.

$$
\boldsymbol{T}+\boldsymbol{T} \alpha(z) \boldsymbol{T}=\frac{\hbar^{2}}{2}\left(\frac{K^{2}}{m_{x y}(z)}-\frac{\partial}{\partial z} \frac{1}{m_{z}(z)} \frac{\partial}{\partial z}\right)
$$

This equation, which is self-adjoint, can be solved for the kinetic energy operator.

$$
\begin{aligned}
\boldsymbol{T} & =\alpha^{-1 / 2} h\left(\boldsymbol{G}_{0}+\boldsymbol{G}_{1} K^{2}\right) \alpha^{-1 / 2} \\
\boldsymbol{G}_{0} & =-\alpha^{1 / 2} \frac{\partial}{\partial z} \frac{\hbar^{2}}{2 m_{z}} \frac{\partial}{\partial z} \alpha^{1 / 2} \\
\boldsymbol{G}_{1} & =\frac{\hbar^{2}}{2} \cdot \frac{\alpha(z)}{m_{x y}(z)} \\
h(x) & =\frac{2 x}{1+\sqrt{1+4 x}}
\end{aligned}
$$

In analogy with (2) the kinetic energy operator has to be decomposed as follows,

$$
\boldsymbol{T}\left(\boldsymbol{G}_{0}+\boldsymbol{G}_{1} K^{2}\right) \approx \boldsymbol{T}_{0}+\boldsymbol{T}_{1} K^{2}+\boldsymbol{T}_{2} K^{4},
$$

where the determination of the operators $\boldsymbol{T}_{i}$ for heterostructures is more complicated than for uniform material parameters since the operators $\boldsymbol{G}_{0}$ and $\boldsymbol{G}_{1}$ no longer commute.

\section{SUBBAND DISPERSION RELATION}

The eigenvalues of (1) at $K=0$ are denoted by $E_{n}^{0}$ and the eigenfunctions by $\zeta_{n}^{0}(z)(n=0,1,2 \ldots)$. The matrix elements of $\boldsymbol{T}_{1}$ and $\boldsymbol{T}_{2}$ in the $\left\{\zeta_{n}^{0}\right\}$ basis are $T_{1, m n}$ and $T_{2, m n}$, respectively. Perturbation theory yields a polynomial representation for $E_{n}(K)$ :

$$
E_{n}(K)=E_{n}^{0}+T_{1, n n} K^{2}+\left(\sum_{m \neq n} \frac{\left|T_{1, m n}\right|^{2}}{E_{n}^{0}-E_{m}^{0}}+T_{2, n n}\right) K^{4}
$$

This expression allows to characterize each subband by an effective mass, $m_{n}$, and a nonparabo- 
licity coefficient, $\alpha_{n}$ defined by

$$
\begin{aligned}
& m_{n}=\frac{\hbar^{2}}{2 T_{1, n n}} \\
& \alpha_{n}=-\frac{1}{T_{1, n n}^{2}}\left(\sum_{m \neq n} \frac{\left|T_{1, m n}\right|^{2}}{E_{n}^{0}-E_{m}^{0}}+T_{2, n n}\right)
\end{aligned}
$$

Although (6) suggests a representation of $E_{n}(K)$ as a polynomial in $K$ it appears favorable to assume a second-order polynomial in the energy instead.

$$
\left(E-E_{n}^{0}\right)\left(1+\alpha_{n}\left(E-E_{n}^{0}\right)\right)=\frac{\hbar^{2} K^{2}}{2 m_{n}}
$$

By comparison with $E_{n}(K)$ obtained from a solution of (1) at discrete $K$-points it was found that (9) gives an excellent approximation for $E_{n}(K)$ in a wide $K$-range, whereas the polynomial (6) is applicable only for sufficiently small $K$.

\section{ELECTRON DENSITY}

The electron density is given as the sum of all position probabilities of the states $|n, K\rangle$ weighted by their occupation probabilities.

$$
n(z)=g_{v} \sum_{n} \sum_{K}\left|\zeta_{n}(z ; K)\right|^{2} f\left(E_{n}(K)\right)
$$

In (10) $f$ denotes the Fermi-Dirac distribution function and $g_{v}$ the valley degeneracy factor. The summation over $K$ is usually converted to an integral by employing the two-dimensional density of states, $\rho_{2 \mathrm{D}, \mathrm{n}}$.

$$
\begin{gathered}
\rho_{2 D, n(E)}=g_{v} \frac{m_{n}}{\pi \hbar^{2}}\left(1+2 \alpha_{n}\left(E-E_{n}^{0}\right)\right) \\
n(z)=\sum_{n} \int_{E_{n}^{0}}^{\infty}\left|\zeta_{n}(z ; K)\right|^{2} f(E) \rho_{2 D, n(E)} \mathrm{d} E
\end{gathered}
$$

The hole density is calculated using Boltzmann statistics. Once the carrier densities are known the electrostatic potential is obtained by solving the one-dimensional Poisson equation. During each self-consistent iteration step the Schrödinger equation and the linearized Poisson equation are solved.

\section{DISCRETIZATION}

The wave functions, which are assumed to vanish at the boundaries, are represented by a Fourier series. After truncation of the series the Schrödinger equation (1) is converted into an algebraic eigenvalue equation [1].

The linearized Poisson equation is discretized in real space using the finite difference method. This yields a tridiagonal equation system which can be efficiently solved. During each iteration step the Fast Fourier Transform is applied to transfer the required quantities from real-space representation to momentum representation and vice versa.

\section{RESULTS AND DISCUSSION}

\subsection{Silicon Inversion Layer}

A MOS capacitor has been simulated. The parameters were chosen as follows: $m_{l}=0.98$, $m_{t}=0.19, \alpha=0.7 \mathrm{eV}^{-1}, t_{\text {ox }}=7 \mathrm{~nm}, N_{A}=5 \cdot 10^{16}$ $\mathrm{cm}^{-3}, V_{G B}=2.5 \mathrm{~V}, T=300 \mathrm{~K}$. A nonparabolic bulk dispersion relation was assumed:

$$
\varepsilon=\frac{2 \gamma}{1+\sqrt{1+4 \alpha \gamma}}, \quad \gamma=\frac{\hbar^{2}}{2}\left(\frac{K^{2}}{m_{x y}}+\frac{k_{z}^{2}}{m_{z}}\right) .
$$

In Figure 1 the Fourier coefficients of the first two wave functions are plotted. This corresponds to a representation of the wave functions in momentum space. The number of harmonics equals $N=64$. Due to nonparabolicity the motion of the carriers normal to the interface is no longer decoupled from the motion parallel to the interface, as is the case for parabolic bands. For nonvanishing $K$ narrower wave functions are observed than for $K=0$. 


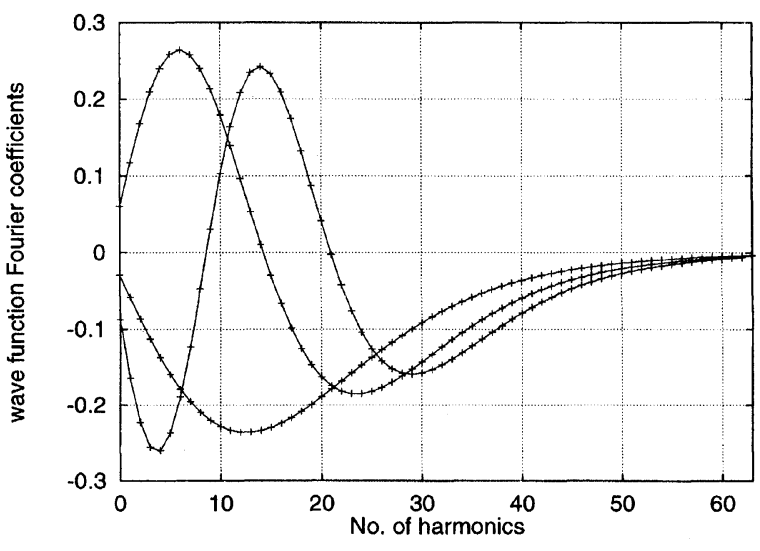

FIGURE 1 Spectrum of the first three wave functions in a silicon inversion layer.

\subsection{InP-Based Pseudomorphic HEMT}

A heterostructure after [2] has been simulated (Tab. I). In the simulation both nonparabolic and parabolic bulk dispersions have been considered. When moving across this heterostructure an electron will experience considerable variations of the bandstructure parameters.

The subband parameters $m_{n}$ and $\alpha_{n}$ are plotted in Figures 2 and 3 for 20 subbands. Strong variations of these parameters can be observed. In the nonparabolic case, the mass increases significantly when going from subband 3 to 4 , while in turn the nonparabolicity coefficient decreases. This means that the changes of $m_{n}$ and $\alpha_{n}$ are correlated. This behavior can be understood when considering the wave functions (Fig. 4). While an electron in subband 3 resides preferably in the InGaAs channel (low mass, high $\alpha$ ), an electron in subband 4 feels predominantly the material properties of the AlInAs barrier.

TABLE I Parameters of the InP heterostructure. The donor layer is doped with $N_{D}=2.10^{18} \mathrm{~cm}^{-3}$

\begin{tabular}{lcccc}
\hline Layer & Material & $\begin{array}{c}t \\
(\mathrm{~nm})\end{array}$ & $\begin{array}{c}\text { mass } \\
\left(\mathrm{m}_{0}\right)\end{array}$ & $\begin{array}{c}\alpha \\
\left(\mathrm{eV}^{-1}\right)\end{array}$ \\
\hline barrier & $\mathrm{Al}_{0.48} \mathrm{In}_{0.52} \mathrm{As}$ & 20 & 0.082 & 0.84 \\
donor & $\mathrm{Al}_{0.48} \mathrm{In}_{0.52} \mathrm{As}$ & 12.5 & 0.082 & 0.84 \\
spacer & $\mathrm{Al}_{0.48} \mathrm{In}_{0.52} \mathrm{As}$ & 2 & 0.082 & 0.84 \\
channel & $\mathrm{In}_{0.53} \mathrm{Ga}_{0.47} \mathrm{As}$ & 20 & 0.038 & 1.02 \\
substrate & $\mathrm{InP}$ & 20 & 0.077 & 0.83 \\
\hline \multicolumn{5}{c}{}
\end{tabular}

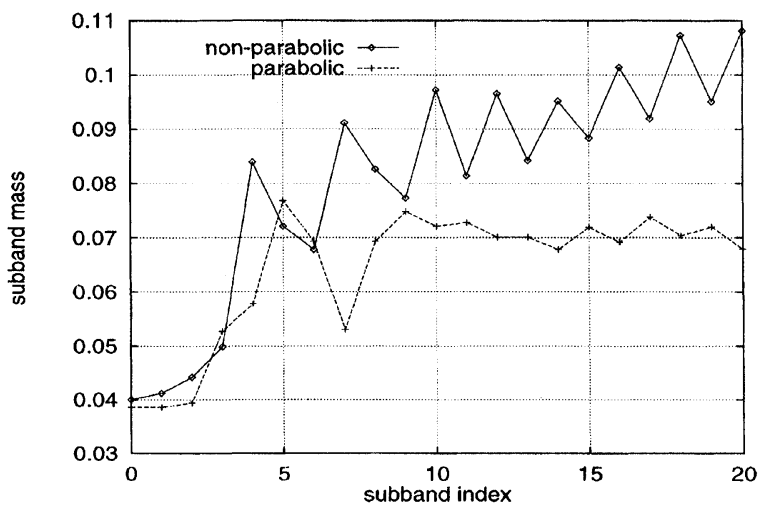

FIGURE 2 The subband masses for the InP heterostructure for both the nonparabolic and parabolic case.

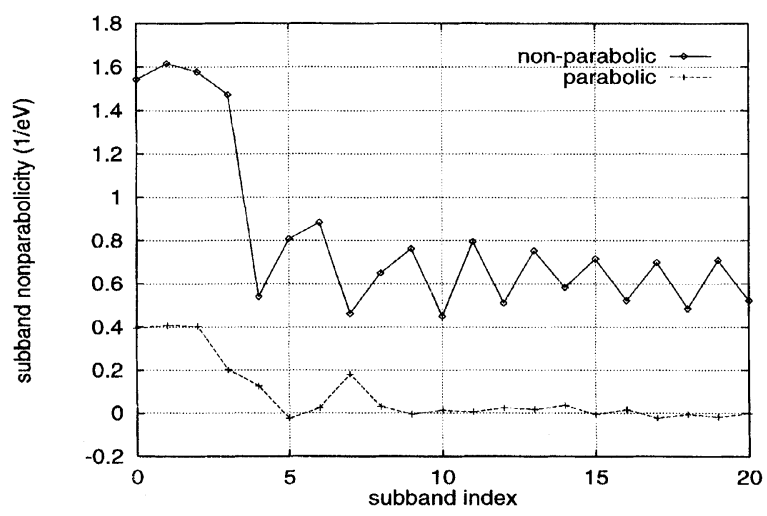

FIGURE 3 The subband nonparabolicity coefficients for the InP heterostructure. Although for the lower curve parabolic bulk dispersions are assumed, the subbands are nonparabolic due to the material inhomogeneity.

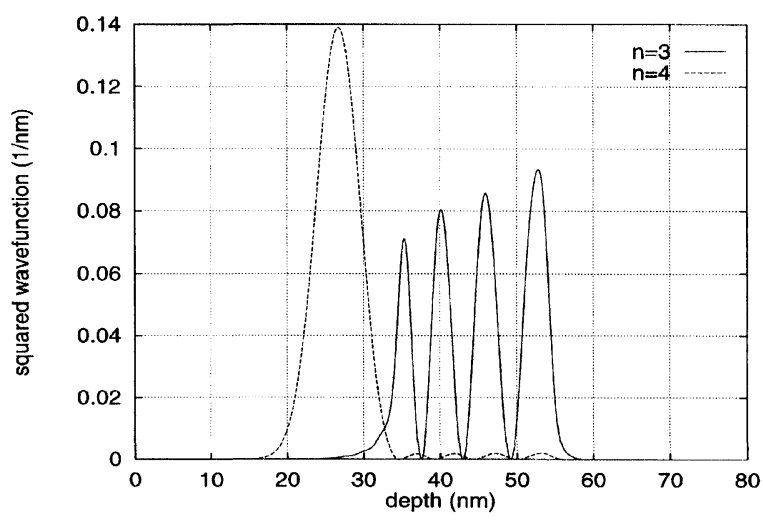

FIGURE 4 Wavefunctions 3 and 4 in the InP heterostructure. 


\section{Acknowledgment}

This work has been supported by the "Fonds zur Förderung der wissenschaftlichen Forschung", Project No. P10642 PHY.

\section{References}

[1] Fischetti, M. and Laux, S. (1993). "Monte Carlo Study of Electron Transport in Silicon Inversion Layers", Physical Review B, 48(4), 2244-2274.

[2] Jungemann, C., Emunds, A. and Engl, W. (1993) "Simulation of Linear and Nonlinear Electron Transport in Homogeneous Silicon Inversion Layers", Solid-State Electron., 36(11), 1529-1540.

[3] Abou-Elnour, A. and Schuenemann, K. (1994). "An Efficient and Accurate Self-Consistent Calculation of Electron States in Modulation Doped Heterostructures", Solid-State Electron., 37 (1), 27-30.

[4] Ali, F. and Gupta, A. (1991). HEMT's and HBT's: Devices, Fabrication and Circuits. Artech House.

\section{Authors' Biographies}

Hans Kosina received the 'Diplomingenieur' degree in electrical engineering and the Ph.D. degree from the Vienna University of Technology in 1987 and 1992, respectively. He was for one year with the 'Institut für flexible Automation' and joined then the 'Institut für Mikroelektronik', where he is currently employed as an Assistant Professor. In summer 1993 he was Visiting Scientist at the Advanced Product Research and Development Laboratory at Motorola Inc., Austin, Texas. His current interests include modeling of hot carrier phenomena and quantum effects in semiconductor devices, and computer aided engineering in VLSI technology.

Christian Troger was born in Vienna, Austria, in 1969. He studied electrical engineering at the Vienna University of Technology, where he received the degree of 'Diplomingenieur' in 1995. He joined the 'Institut für Mikroelektronik' in September 1995, where he is currently working for his doctoral degree. His work is focused on the simulation of quantum mechanical devices. 

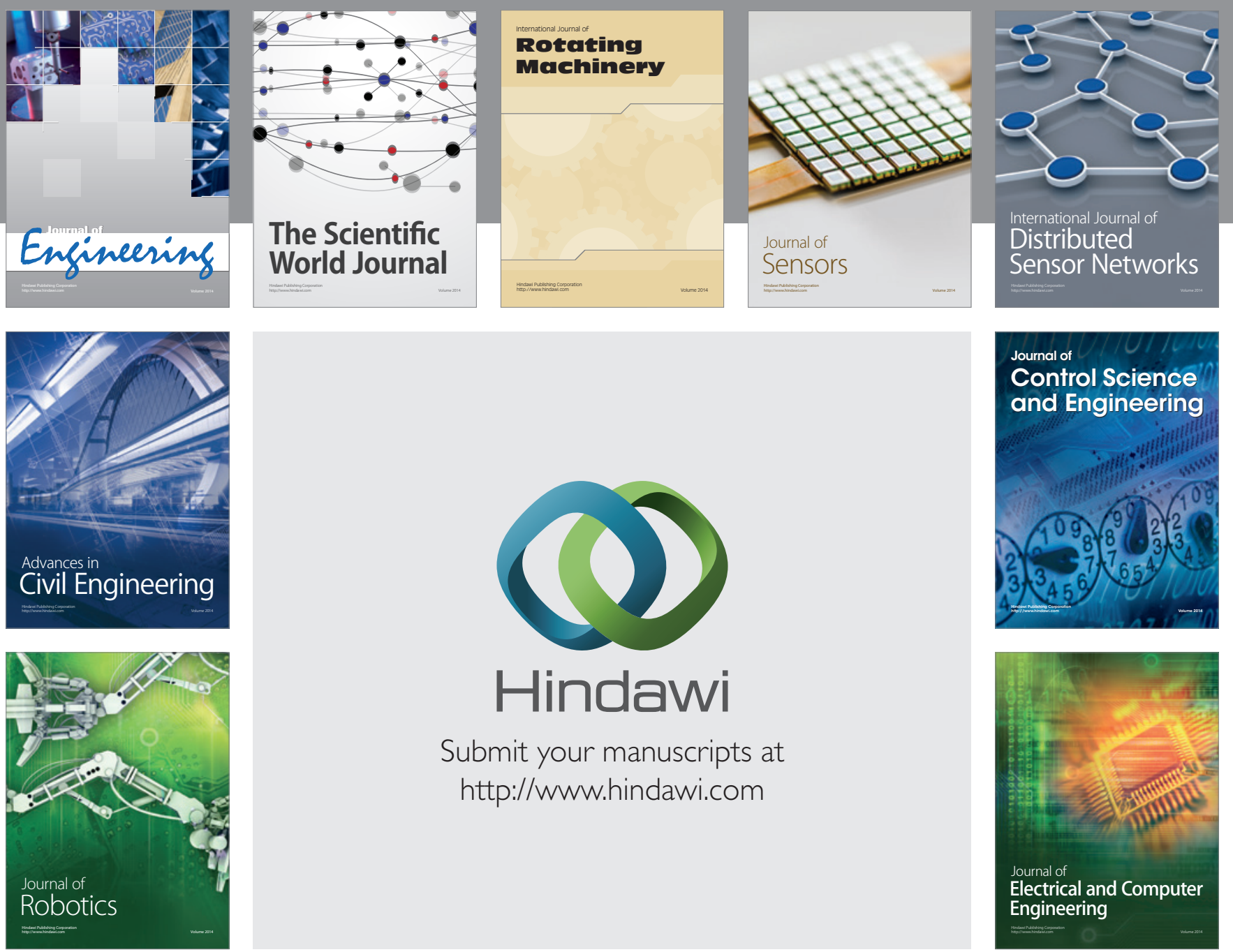

Submit your manuscripts at

http://www.hindawi.com
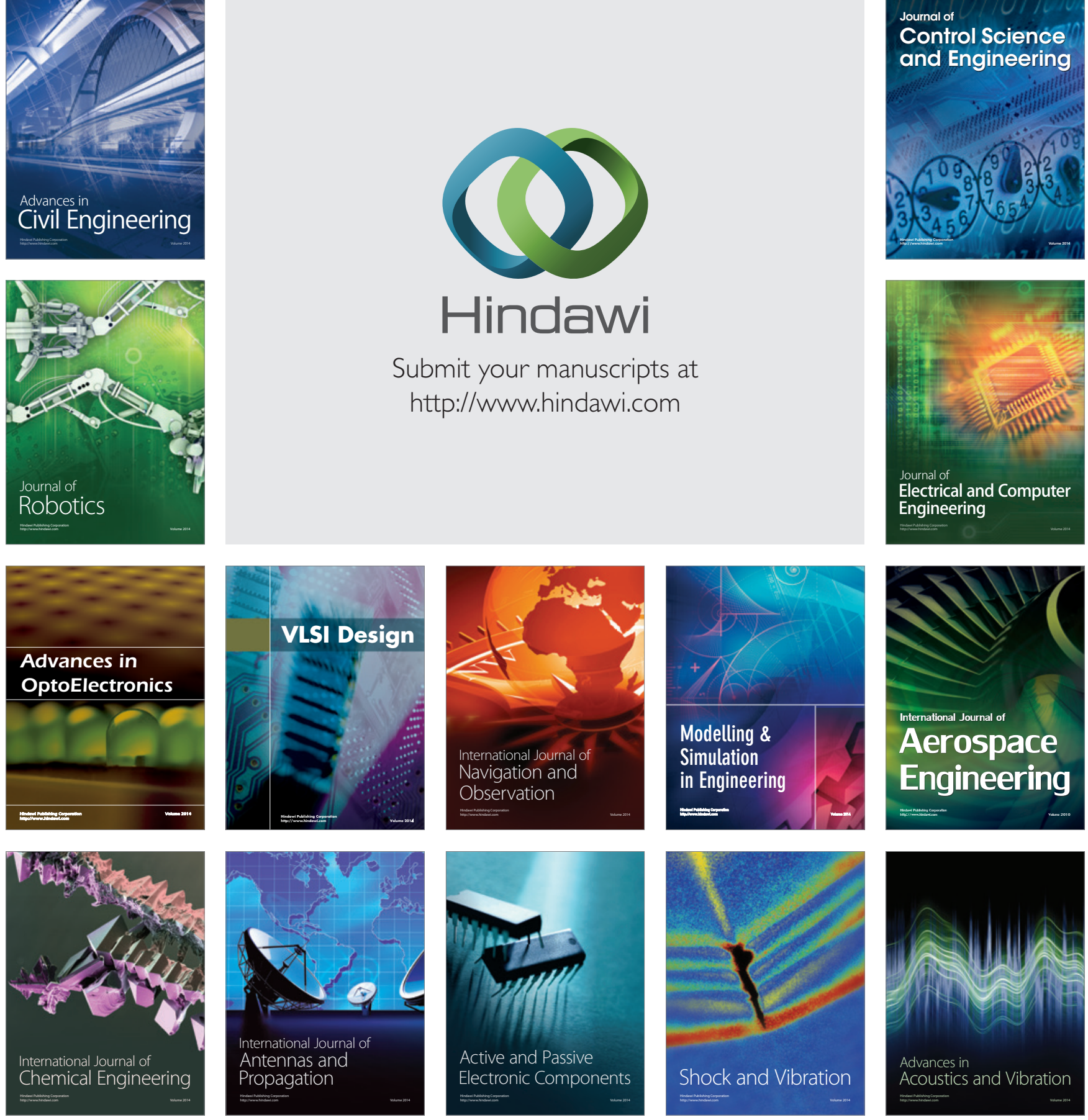